\title{
II faut réévaluer la place de l'adaptation dans la politique climatique
}

\author{
Michel Damian \\ Économiste, LEPII, Université Pierre Mendès France / CNRS, BP 47, 38040 Grenoble cedex 9, France
}

Les réponses au changement climatique ont négligé les enjeux relatifs à l'adaptation. Cette position n'est plus tenable une fois admise l'inéluctabilité du réchauffement. Reconnaître les besoins d'adaptation contribuerait à mieux faire accepter le renforcement des politiques de réduction des émissions de gaz à effet de serre.

Les réflexions et les travaux en cours sur la politique climatique française devraient accorder toute l'attention qu'elles méritent aux politiques d'adaptation aux impacts du réchauffement, et ce, pour au moins trois raisons : 1) la thématique est à l'ordre du jour de l'agenda diplomatique international : l'adaptation et son financement - et non plus uniquement les politiques d'atténuation - seront au centre des négociations sur la politique post-Kyoto ; 2) elle n'est plus, comme par le passé, à la marge des préoccupations : la place de l'adaptation dans la politique climatique à été minorée au cours de la dernière décennie, sinon depuis les premières négociations ; or, cette position n'est plus aujourd'hui tenable ; 3 ) le réchauffement climatique et l'adaptation sont inévitables : autant le reconnaître et en tirer toutes les conséquences ${ }^{1}$.

\section{L'adaptation est à l'ordre du jour de la politique climatique}

La conférence de Nairobi sur le changement climatique, en novembre 2006, marque un tournant dans l'attention accordée à la politique d'adaptation. Kofi Annan, alors secrétaire général des Nations unies, a lancé un appel pour des mesures urgentes d'adaptation au bénéfice des pays pauvres. Le Bangladesh, au nom du groupe des pays les moins avancés, a proposé l'instauration d'une taxe internationale sur le transport aérien (les luxury

\footnotetext{
Auteur correspondant : michel.damian@upmf-grenoble.fr

1 Mes remerciements à Danièle Revel, Nathalie Rousset, Patrick Criqui et Jean-Christophe Graz.
}

emissions, selon la formulation d'Anil Agarwal) pour financer l'adaptation. Le président de la Confédération suisse Moritz Leuenberger a, lui, souhaité une taxe $\mathrm{CO}_{2}$ mondiale pour financer l'adaptation et réparer, selon ses termes, «l'injustice du changement climatique », les plus touchés étant ceux qui y ont le moins contribué jusqu'ici. Cette conférence n'a abouti, selon les pays et les médias occidentaux, à aucun résultat tangible ni aucune orientation pour l'action future, mais elle a été vécue par les pays en développement comme une avancée de par l'accent mis sur l'adaptation, son financement et la gestion de celui-ci ${ }^{2}$.

La thématique de l'adaptation a depuis envahi l'agenda international. Georges W. Bush, dans sa déclaration du 31 mai 2007, peu avant le sommet du G8 de Heiligendamn, a présenté plusieurs axes de travail pour un nouvel accord sous l'égide de la Convention-cadre des Nations unies sur les changements climatiques de 1992, proposant en particulier de développer des technologies plus efficientes et plus propres - parce qu'en matière de technologie, "United States is in the lead»-, mais en mettant en tête l'adaptation au changement climatique. Cette ligne d'action avait été précédée de prises de position similaires en provenance de toutes les grandes organisations internationales. En janvier 2007, le Programme des Nations unies pour le développement (PNUD) annonçait que son prochain Rapport mondial sur le développement humain (publié en novembre sous le titre La lutte contre le changement climatique : un impératif de solidarité humaine dans un monde divisé) traiterait en particulier de l'adaptation. Le 13 mars, Paul Wolfowitz,

\footnotetext{
${ }^{2}$ Cf. Müller, B., 2007. The Nairobi Climate Change Conference: a breakthrough for adaptation funding, Oxford Energy $\mathcal{E}$ Environment Comment, January; Okereke, C., et al., 2007. Assessment of key negotiating issues at Nairobi climate COP/MOP and what it means for the future of the climate regime, Tyndall Centre Working Paper, 106.
} 
alors président de la Banque mondiale, déclarait depuis Londres que l'adaptation est le problème des pays pauvres et que la Banque devait accorder beaucoup plus d'attention à l'adaptation dans son travail quotidien. Le 6 avril, suite à la présentation à Bruxelles du rapport du groupe de travail II du GIEC (Groupe d'experts intergouvernemental sur l'évolution du climat), Bilan 2007 des changements climatiques : impacts, adaptation et vulnérabilité, le secrétaire général de la Convention-cadre sur les changements climatiques, Ivo de Boer, insistait sur le besoin urgent de financement pour l'adaptation : les sources actuelles sont insuffisantes. Le 10 du même mois, Achim Steiner, directeur du Programme des Nations unies pour l'environnement (PNUE), déclarait depuis Bangkok, à l'issue de la présentation du rapport du groupe de travail III du GIEC, que l'action ne peut plus être confinée à la réduction des émissions, parce que le changement climatique est inévitable.

Les règles de l'actuel Protocole de Kyoto seront caduques en décembre 2012. Les négociations post-Kyoto ont débuté en décembre 2007, à Bali, lors de la conférence sur le changement climatique. Trois au moins des grandes lignes directrices de la prochaine scène climatique sont déjà connues ${ }^{3}: 1$ ) $1^{\prime} U E$ devra conduire une rude bataille pour définir de nouveaux objectifs quantifiés de réduction, tenter de renforcer le Protocole et l'élargir à de nouveaux pays ; 2) les négociations les plus ouvertes se dérouleront aux Nations unies, dans le contexte institutionnel de la Convention-cadre sur les changements climatiques (Kyoto étant un protocole à la Convention), où de nombreuses options devraient être "sur la table», selon les termes du secrétaire général de la Convention ; 3) l'adaptation au changement climatique et son financement - et non plus uniquement les politiques d'atténuation - seront au centre des débats. L'UE, qui porte le Protocole depuis son origine, a été jusqu'à présent silencieuse sur ces orientations.

\section{L'importance de l'adaptation a été minorée}

La Convention-cadre négociée à Rio se réfère à plusieurs reprises à l'adaptation et à la vulnérabilité, et l'adaptation est devenue un enjeu climatique et politique de plus en plus intense lors des récentes conférences sur le changement climatique ${ }^{4}$. Mais la question de l'adaptation, longtemps demeurée un tabou, a été maintenue à la lisière des débats ${ }^{5}$. Elle empoisonne en effet les négociations

\footnotetext{
${ }^{3}$ Damian, M., Abbas, M., 2007. Politique climatique et politique commerciale : le projet français de taxe $\mathrm{CO}_{2}$ aux frontières de l'Europe, Revue de l'énergie, 578, 221-230.

4 Schipper, E.L., 2006. Conceptual history of adaptation in the UNFCCC process, Review of European Community E International Environmental Law, 15, 1, 82-92.

${ }^{5}$ Cf. Pielke, Jr., R.A., 1998. Rethinking the role of adaptation in climate policy, Global Environmental Change, 8, 2, 159-170;
}

depuis les premiers travaux du GIEC, à la fin de la décennie quatre-vingt. Cinq raisons, difficiles à hiérarchiser, permettent de comprendre pourquoi il y a eu un tel biais de la politique climatique à l'encontre de l'adaptation.

Premièrement, parler d'adaptation - « une idée inacceptable et même politiquement incorrecte ${ }^{6} »-$, cétait $^{\prime}$ anticiper l'échec de la lutte contre les émissions de gaz à effet de serre : impensable. Deuxièmement, l'adaptation concernant prioritairement les pays en développement aux faibles moyens et/ou aux conditions climatiques déjà difficiles, la thématique ouvrait la porte aux revendications de compensations financières de pays qui n'étaient pas les responsables majeurs du changement climatique : un débat politique que tous les grands émetteurs historiques de $\mathrm{CO}_{2}$ ont longtemps tenté d'éluder. Troisièmement, l'adaptation apparaissait indissociable des processus de développement et de l'évolution des sociétés, difficile à définir, avec des coûts impossibles à évaluer précisément et sans outil explicite pour la mise en œuvre de politiques, alors que l'atténuation pouvait être rendue plus immédiatement opérationnelle, avec un objectif (réduire les émissions de $\mathrm{CO}_{2}$ ), des outils (ceux de l'écologie de marché : permis négociables et/ou taxes) et un résultat attendu (éviter le réchauffement). Quatrièmement, même si la reconnaissance par certains experts du caractère peut-être irréversible du changement climatique ${ }^{7}$, et donc inévitable de l'adaptation ${ }^{8}$, est ancienne, les incertitudes étaient alors grandes : il a fallu attendre le quatrième rapport du GIEC, en 2007, pour que les scientifiques affirment, d'une part, que le climat était en train de changer plus rapidement que précédemment anticipé et que, d'autre part, le réchauffement persisterait pendant des siècles ${ }^{9}$.

Il y a enfin une cinquième et dernière raison qui permet de comprendre pourquoi il demeure difficile de parler d'adaptation, celle de la définition même du changement climatique dans l'article 2 de la Convention. Cet article, très discuté dans les cercles de scientifiques et $\mathrm{d}^{\prime}$ experts, a une longue histoire ${ }^{10}$. Il explicite « l'objectif ultime » de la Convention et de toutes les politiques susceptibles d'être mises en œuvre dans la formulation

Pielke, Jr., R., et al., 2007. Lifting the taboo on adaptation, Nature, 445, 597-598.

6 Burton, I., 1994. Deconstructing adaptation... and reconstructing, Delta, 5, 1, 14.

7 Cooper, C.F., 1978. What might man-induced climate change mean?, Foreign Affairs, 56, 3, 500-520.

8 Cf. Parry, M., et al., 1998. Adapting to the inevitable, Nature, 395, 741 ; Rayner, S., Malone, E.L., 1997. Zen and the art of climate maintenance, Nature, 390, 332-334.

9 Groupe d'experts intergouvernemental sur l'évolution du climat (GIEC), Groupe de travail I, 2007. Bilan 2007 des changements climatiques : les bases scientifiques. Résumé à l'intention des décideurs, 2 février, Paris ; $c f$. p. 16-17.

10 Voir l'analyse érudite de Oppenheimer, M., Petsonk, A., 2005. Article 2 of the UNFCCC: historical origins, recent interpretations, Climatic Change, 7, 3, 195-226. 
suivante : «stabiliser [...] les concentrations de gaz à effet de serre dans l'atmosphère à un niveau qui empêche toute perturbation anthropique dangereuse du système climatique. Il conviendra d'atteindre ce niveau dans un délai suffisant pour que les écosystèmes puissent s'adapter naturellement aux changements climatiques ».

La Convention définit le changement climatique en référence au forçage radiatif des gaz à effet de serre, l'adaptation étant supposée se réaliser «naturellement». Le GIEC, lui, a une appréhension plus large, incluant non seulement les changements engendrés par les activités humaines, mais également ceux dus à la variabilité naturelle du climat - variabilité à laquelle les sociétés ont toujours eu, du moins à plusieurs reprises, à s'adapter. La manière dont la Convention conçoit le changement climatique a eu deux conséquences : 1) la politique menée sous l'égide de la Convention a été entièrement focalisée sur le $\mathrm{CO}_{2}$ et la politique énergétique ; 2 ) l'adaptation est demeurée en quelque sorte hors du champ de vision, la politique climatique de la dernière décennie, et même des vingt dernières années, témoignant d'un biais à l'encontre de $l^{\prime}$ adaptation ${ }^{11}$. Une telle position n'est plus aujourd'hui tenable.

\section{Le réchauffement climatique et l'adaptation sont inéluctables}

L'article 2 de la Convention a influencé, voire est à l'origine du concept de «safe corridor ${ }^{12}$ ", c'est-à-dire de l'idée de trajectoires d'émissions susceptibles de stabiliser les concentrations à long terme et ainsi de limiter le réchauffement à un niveau acceptable. Cette idée de trajectoire fonde l'objectif de l'UE des 2 degrés à ne pas dépasser sur le long terme, avec des réductions d'émissions escomptées de 20 à $30 \%$ d'ici 2020 pour les pays européens. Pour demeurer dans la ligne des 2 degrés au plan mondial à l'horizon 2050, une obligation de réduction globale allant bien au-delà de $50 \%$ par rapport au niveau de 1990 serait nécessaire, avec un pic des émissions mondiales au plus tard autour de $2015^{13}$. Les derniers travaux du GIEC, son quatrième rapport, montrent cependant que les réductions envisagées par l'UE pour les pays industrialisés, déjà extrêmement ambitieuses, ne sont plus en phase avec l'objectif des 2 degrés. L'emballement des émissions mondiales de $\mathrm{CO}_{2}$ au cours des premières années de ce siècle $\mathrm{e}^{14}$

\footnotetext{
11 Sur ces points, voir les contributions déterminantes de Pielke, Jr., R.A., 1998, loc. cit. ; Pielke, Jr., R.A., 2005. Misdefining “climate change": consequences for science and action, Environmental Science \& Policy, 8, 548-561 ; voir également Rayner, S., Malone, E.L., 1997, loc. cit.

12 Oppenheimer, M., Petsonk, A., 2005, loc. cit., p. 204.

13 Den Elzen, M., Meinshausen, M., 2006. Meeting the UE $2{ }^{\circ} \mathrm{C}$ climate target: global and regional emission implications, Climate Policy, 6, 5, 545-564.

14 Raupach, M.R., et al., 2007. Global and regional drivers of accelerating $\mathrm{CO}_{2}$ emissions, PNAS, in Proceedings of the National
}

rend encore moins tenable le niveau de réchauffement prôné par l'UE.

Jusqu'à la période récente, les initiatives et les prises de position de l'UE sur l'adaptation étaient demeurées limitées et fragmentées ${ }^{15}$. Un pas vient cependant d'être franchi avec la publication, en juin 2007, du Livre vert de la Commission européenne sur l'adaptation : le changement climatique est «inéluctable [...] même si les efforts d'atténuation déployés à l'échelle de la planète au cours des prochaines décennies portent leurs fruits »; l'adaptation est donc «inévitable», seule "une réduction immédiate et drastique des émissions permettrait d'éviter de trop graves répercussions ${ }^{16}$.

Le rapport sur l'économie du changement climatique publié par Nicholas Stern à l'automne $2006^{17}$, à la demande du chancelier de l'Échiquier britannique, a été remarqué et célébré. Il a aussi été lu sous le prisme critique d'économistes du monde anglo-américain. Ian Burton - auteur principal du groupe de travail II du GIEC - l'a lu, lui, avec l'œil du spécialiste reconnu qu'il est de l'adaptation. Voici ce qu'il écrit :

«Les coûts de l'adaptation sont principalement discutés pour montrer qu'ils vont croître rapidement sans politique d'atténuation, et non pour soutenir la nécessité immédiate de politiques d'adaptation. Stern affirme également que "l'adaptation est complexe et que de nombreux obstacles restent à surmonter", et que "même avec des politiques publiques appropriées, l'adaptation sera contrainte à la fois par l'incertitude et par des limites techniques". Hé là! Qui peut croire que l'atténuation est vraiment simple à mettre en œuvre et ne comporte pas de limites techniques? [...] Stern échoue à donner à l'adaptation le vigoureux coup de main dont elle a besoin ${ }^{18}$.»

N. Stern a depuis accordé publiquement son soutien au récent rapport d'Oxfam, Adapting to Climate Change ${ }^{19}$.

Academy of Sciences of the United States of America. Disponible sur: http://www.globalcarbonproject.org/global/pdf/pep/Post2006/ Raupachetal.2007.CO2Emissions.PNAS.All.pdf (consulté le 20/08/2007).

15 Yamin, F., 2005. The European Union and future climate policy: is mainstreaming adaptation a distraction or part of the solution?, Climate Policy, 5, 349-361.

${ }^{16}$ Commission des Communautés européennes, 2007. Adaptation au changement climatique en Europe : les possibilités d'action de l'Union européenne, Livre vert, COM(2007) 354 final, Bruxelles, 29 juin, p. 3.

17 Stern, N. (Ed.), 2006. The Stern Review Report: The Economics of Climate Change, London, HM Treasury.

18 Burton, I., 2007. Modelling Adaptation?, Tiempo Climate Newswatch, 62. Disponible sur : http://www.tiempocyberclimate. org/newswatch/xp_comment070212.htm

(consulté le 20/08/2007).

19 Oxfam International, 2007. Adapting to Climate Change: What's Needed in Poor Countries, and Who Should Pay, Oxfam 
L'adaptation - que ce soit à New York, Londres ou Bruxelles - n'est maintenant plus à la lisière de la politique climatique.

Pour les spécialistes les plus interrogatifs à l'égard de l'action des quinze dernières années, il ne suffit cependant pas de lever à la marge le tabou de l'adaptation, il faut complètement réévaluer la place de celle-ci dans les politiques climatique et de développement soutenable:

«Tant que l'adaptation est discutée en termes d'effets marginaux sur le changement climatique anthropique, son importance réelle pour la société est masquée [...]. Mais le fait de définir l'adaptation en termes de développement soutenable s'ajuste mal au cadre politique au sein duquel est actuellement posé le problème du changement climatique. En introduisant la dimension du développement soutenable, on est contraint de constater les opportunités manquées d'un régime international qui, au cours des quinze dernières années ou plus, a concentré d'énormes ressources intellectuelles, politiques, diplomatiques et fiscales sur l'atténuation, tout en minorant l'importance de l'adaptation. Tant que l'adaptation ne sera pas prise en compte au plan institutionnel à un niveau d'intensité et d'investissement au moins égal à ceux de la Convention-cadre sur les changements climatiques et du Protocole de Kyoto, les impacts du climat continueront à augmenter sans être limités, même dans le cas de réductions très significatives des émissions de gaz à effet de serre ${ }^{20}$. »

\section{Conclusion}

En octobre 1985, à Villach (Autriche), se tenait la première grande conférence sur le $\mathrm{CO}_{2}$ et l'effet de serre, une conférence emblématique, puisque les scientifiques présents soutinrent pour la première fois que le réchauffement climatique était « hautement probable ${ }^{21}$ ». Peu après cette conférence, le PNUE publiait le premier de ses dossiers sur l'environnement, avec pour titre : L'Atmosphère en mutation. Ces dossiers sont édités «pour éveiller l'attention du public sur les questions écologiques globales $»^{22}$.

Briefing Paper, May. Disponible sur :

http://www.oxfam.org.uk/what_we_do/issues/climate_change/ downloads/bp104_adapting_to_climate_change.pdf (consulté le 20/08/2007).

20 Pielke, Jr., R., et al., 2007, loc. cit., p. 598.

21 World Climate Programme, World Meteorological Organization (WMO), 1986. Report of the International Conference on the Assessment of the Role of Carbon Dioxide and Other Greenhouse Gases in Climate Variations and Associated Impacts (Villach, Austria, 9-15 October 1985). Doc. $\mathrm{n}^{\circ}$ 661, p. 57.

22 Programme des Nations unies sur l'environnement (PNUE), 1986. L'Atmosphère en mutation, Dossier sur l'environnement, 1 ;
Trois décennies plus tard, la certitude «globale » est que le réchauffement a commencé, qu'il se poursuivra sur plusieurs siècles, l'adaptation aussi.

Les lignes qui précèdent ne visent cependant pas à minimiser l'urgence de politiques fortes de réduction des émissions - ce à quoi conduit la position des économistes anglo-américains (en particulier Richard Tol, William Nordhaus, Scott Barrett et Thomas Schelling), lorsqu'ils soutiennent que les générations présentes peuvent assumer les coûts actuels de l'adaptation, encore faibles, alors que les générations futures, supposées devenir plus riches, seraient susceptibles d'absorber des coûts d'adaptation plus élevés. Olivier Godard a produit une analyse critique serrée de ces travaux. Il reconnaît qu'il n'est « pas contestable que toute politique de l'effet de serre doit intégrer des mesures visant à faciliter l'adaptation des personnes, de la production et des sociétés ${ }^{23} »$. Mais est-il bien judicieux d'écrire que « la logique ultime de la rhétorique de l'adaptation à un environnement bouleversé est d'approfondir la dissociation entre la vie humaine et le fonctionnement de la biosphère » ou encore que "l'existence de stratégies d'adaptation pousse à l'accélération rationnelle du processus de dégradation environnementale ${ }^{24}$ ?

Cette thématique de l'adaptation a été jusqu'à présent trop discrète en France, du moins dans les travaux économiques et à orientation écologique : le terme «adaptation » ne figure pas dans le rapport du Conseil d'analyse économique sur «l'économie de l'effet de serre » dirigé par Roger Guesnerie ${ }^{25}$, ni d'ailleurs dans le Pacte écologique de Nicolas Hulot ${ }^{26}$. Puisque le changement climatique est « inéluctable », il faut le dire, en débattre largement, s'y préparer. Reconnaître l'effet quasiment indiscernable de Kyoto à terme, l'impossibilité des politiques post-Kyoto même les plus volontaristes - à empêcher le changement climatique et donc la nécessité de l'adaptation, c'est certainement un choc narcissique. C'est même le signal le plus fort qui puisse être donné pour crédibiliser, mieux faire accepter, l'indispensable renforcement des politiques d'atténuation des émissions de gaz à effet de serre.

Programme des Nations unies sur l'environnement (PNUE), 1986. Quand l'effet de serre menace..., Nouvelles du PNUE, marsavril.

${ }^{23}$ Godard, O., 2007. Climat et générations futures : un examen critique du débat académique suscité par le Rapport Stern, École polytechnique, Cahier de la chaire du Développement durable, DDX07-12, p. 21.

24 Ibid., p. 26.

25 Guesnerie, R. (Ed.), 2002. L'Économie de l'effet de serre, Paris, La Documentation française.

${ }^{26}$ Hulot, N., 2006. Pour un pacte écologique, Paris, CalmannLévy. 